\title{
Publisher's Note: Enhanced caloric effect induced by magnetoelastic coupling in NiMnGaCu Heusler alloys: Experimental study and theoretical analysis [Phys. Rev. B 96, 224105 (2017)]
}

Dewei Zhao, Teresa Castán, Antoni Planes, Zongbin Li, Wen Sun, and Jian Liu

Q (Received 22 January 2018; published 25 January 2018)

DOI: 10.1103/PhysRevB.97.019907

This paper was published online on 27 December 2017 with an error in the author affiliations. The third author affiliation should read as " ${ }^{3}$ Departament de Física de la Matèria Condensada, Facultat de Física, Universitat de Barcelona, Diagonal 647, 08028 Barcelona, Catalonia, Spain." The affiliation has been corrected as of 22 January 2018. The affiliation is incorrect in the printed version of the journal. 